\title{
Beiträge zur Kolloidchemie des Brotes, II.
}

\section{Zur Viskosimetrie der Mehle.}

Von Heinrich Lüers (München) und Wolfgang Ostwald (Leipzig). (Aus der Deutschen Forschungsanstalt für Lebensmittelchemie in München.)

1. Ein le it u ng. In einer vorangegangenen Arbeit" wurde u. a. darauf hingewiesen, daB neben der bisher vorwiegend ausgeübten analytisch - chemischen Untersuchung der Backmaterialien und des Backvorgariges auch eine kolloidchemische Kennzeichnung derselben zweckmäbig erscheint. Der Hauptgrund tür den Vorschlag, auch kolloidchemische Gesichtspunkte und Methoden hier heranzuziehen, war die Erkenntnis, daB Backmaterialien und Backprodukte Stoffe und Gebilde von vorwiegend kolloider Beschaffenheit sind, und daB daher definitionsgemä Kolloiderscheinungen in fast allen Stadien der Brotbereitung eine wichtige Rolle spielen müssen.

$\mathrm{Zu}$ den Problemen, die sich für eine kolloidchemische Bearbeitung besonders emptahlen, gehört auch die viel erörterte Frage nach einer Laboratoriumsmethode zur Kennzeichnung der sog. Backfähigeit der Mehle. Auch hier hat die Praxis erkannt, daB die chemisch-analytische Prüfung der Mehle zwar wichtige Hinweise geben kann, allein indessen nicht genügt, um ein Mehl in backtechnischer Hinsicht $z \mathfrak{u}$ kennzeichnen. Aus dieser sowie aus der anderen Erkeuntmis heraus, dal die physikal is chen Eigenschaften der Mehle nicht minder wichtig sind als ihre chemischen, hat die Praxis mehrfach versucht, physikalisch-chemische Apparate und Methoden zur Kennzeichnung der Mehle zu erfinden, anscheinend aber noch nicht mit durchschlagendem Ertolg 7 .

Es ist nun in der zit. Arbeit bereits ausgefuhrt worden, wie ganz ahnliche Probleme auch in anderen Kolloddindustrien, z. B. in denen des Kautschuks, der Nitrozellulose, der Viskose usw. vorliegen, Probleme, bei denen es sich ebenfalls meist darum handelt, eine Laboratoriumsmethode zu finden, die einen Hinweis aut die zu erwartenden physikalischen Eigenschaften des technischen Endproduiktes erlaubt. In vielen Fallen hat sich dabei herausgestell, dab die Eigenschaften der meist als Qele aufiretenden Endprodukte erschlossen

W Wo. Ostwid, Koll-Zelischr. 8 ,

Siehe die loc. cit. zidlete Literatur. werden können schon aus den Eigenschaften ihrer verdiunnten Sole. Insbesondere aber stehen die mechan is $\mathrm{ch}$ en Eigenschaften der Gele, wie ZerreiBfestigkeit, Elastizität usw., in engem, z. T. überraschend einfachen Zusammenhang mit einer bequem meBbaren Eigenschaft flüssiger Sole, ihrer Visk os ität. Es wurde daher vorgeschlagen, zu versuchen, ob sich

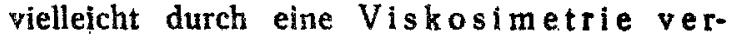
dünnter Mehl-Wasser-Gemische das Problem der Backfähigkeit der Mehle auf kolloidchemischem Wege angreifen lasse.

lm Folgenden seien nun einige experimentelle Beiträge zu dieser Frage mitgeteilt. Die Verfasser sind weit von der Meinung entfernt, mit den bisher erbaltenen Resultaten das fragliche Problem gleich im ersten Anlauf gelöst zu haben. Wohl aber meinen sie, dab die bisherigen Ergebnisse zeigen, dab auf diesem Wege tatsächlich neue, d. h. bisher im Laboratorium unbekannte Eigenschaften von Mehlen aufgezeigt. und studiert werden können, und dab weiterhin die gefundenen Resultate in engem $Z$ us am men hangemitder Eigenschaft der Backfähigkeit stehen. Es ist klar, dab nur auf Grund eines auperst urnfang reichen, systematisch gesammelten Versuchsmaterials Schlüsse gezogen werden können, die unmittelbar auf die Praxis übertragbar' sind. Ein Hauptziel vorliegender Mitteilung ist daher drittens, den Pachgenossen auf båckereiwissen. schaftichem Gebiete darzutun, dak auf dem eingeschlagenen Wege Fortschritte zu erzielen sind, und sie damit anzuregen, selbst in dieser Richtung zu arbeiten.

2. Kennzeichnung der Mehle bei der Teigbereitung und Kennzeichnung derselben beim eigentlichen B a ckprozeB. Die unter dem Namen „Backfähigkeit" zusammengefaßte Gruppe physikalischchemischer Eigenschaften der Mehle aubert sich bekanntlich nicht nur beim eigentlichen Backprozef, sondern schon bei dem vorhergehenden Vorgang der Teigbereitung. Ein gut backfähiges Mehl gibt eine hohe Teigausbeute, einen gut auf Gare stehenden Teig usw. Eine kolloidchemische 2 . B. viskosimetrische Untersuchung hätte sich also sowohl auf die Eigen- 
schaften von Teiglösungen zu erstrecken als auch auf diejenigen von Mehl-WasserGernischen, die durch Erhitzen von Teiglosungen entstehen, m. a. W. auf Mehlkleister-Lösungen. Denn man kann zweifellos das Erhitzen einer verdünnten Teiglösung z. B. während einer halben Stunde auf $100^{\circ}$ ansehen als einen dem Backvorgang in erster Annäherung analogen ProzeB. Es rer. kleistert hierbel nicht nur die Stärke, sondern gleichzeitig findet eine Gerinnung der Mehleiweikkörper statt, eine Zunahme „löslicher Polysaccharide" ist analog wie beim BackprozeB auch hier sehr wahrscheinlich usw. Natürlich entsprechen solche erhitzte Teiglösungen ("flüssiges Brot") nur der Gerüstsubstanz des Brotgel-Schwammes; nicht jedoch dem dispersen System höherer Ordnung selbst, wie es das Brot mit seiner dispersen Gasphase usw. im Ganzen darstellt. Nur auf die physikalischchemischen Eigenschaften dieser Gerüstsubstanz können dementsprechend Schlüsse aus den Eigenschaften des flüssigen Mehlkleisters gezogen werden.

Es folgt aus diesen Ueberlegungen, dab sich zunächst zwei große Gebiete von Viskositatsmessungen anbieten, von denen das eine sich auf die Kennzeichnung der Mehle bei der Teigbereitung, das andere auf die Kennzeichnung derselben beim B a ckvorgang beziehen würde. Es ist dabei keineswegs notwendig, ja aicht einmal $z u$ erwarten, dab die Resultate der Viskosimetrie der Teigbereitung unmittelbare Schlüsse gestatten auch auf die Eignung der Mehle für dea Back proze日. Denn die Physik der Teigbereitung ist $z$ welfellos nicht identisch mit der Physik des Backvorganges selbst, wennschon natürlich enge, aber eben noch zu erforschende $\mathrm{Zu}$ sammenhänge zwischen den physikalischchemischen Eigenschaften des Teiges und denen des tertigen Gebäckes bestehen. Die Verfasser möchten aber mit Nachdruck darauf hinweisen, dat es sich zunächst um $z$ wei versch ie dene, selbsiăndig zu behandelnde Gruppen technischer Prozesse handelt, und daf sichere Schlüsse aus den Eigenschaften des Teiges auf diejenigen des Gebäckes cerst dann zu erwarten sind, wenn beide Gebiete zunächst unabhängig von einander näher studiert worden siad. Vermutich hangt die grobe Unklarheit; die dem Begriffe der "Backfähigkeit" der Mehle der Literatur nach anhaftet, zu einem Teile damit zisammen, das aus dem z. B. günstigen Verbajten der Mehle bei der Teigbereitung vielfuch ohne weiteres auch Schliusse auf ein entsprechendes Resultat beim Backvorgang selbst gezogen wurden. Nicht nur theoretisch, sondern auch praktisch ergibt aber ein technisch einwandfreier Teig keineswegs notwendig auch ein technisch gleich vollkommenes Backprodukt.

3. ZurVersuchs methodik. Wăhrend der eine kolloide Hauptbestandteil der Mehle, die Stärke, bekanntlich mehrfach kolloidchem und auch viskosimetrisch untersucht wurde ${ }^{3}$ ), liegen entsprechende Arbeiten aber dje backtechnisch mindestens ebenso wichtigen Eiweibstoffe des Brotes bisher nicht vor. Wären wir aber auch uber die Viskosimetrie z. B. des reinen Gliadins und des reinen (iutenins') ebenso unterrichtet wie über die der Stärlke, so wäre es vermutlich doch mur sebr schwierig möglich, sichere und quantitative Schiusse auf das Verhalten der Gemische zu ziehen, wie sie im natürlichen Mehl vorliegen. Es ist zweifellos leichter, ein Mekl aus seinen isolierten Bestandteilen künstlich so zusammenzusetzen, dat es in analytisch-chemischer Hinsicht dem natür lichen entspricht, als wenn man diesen Versuch auch auf eine Synthese der kolloidghysikalischen Eigenschaften des Mekles ausdehnen wollte. Vom. Standpunkte der Praxis aus, die hier allein das Problem stellt und kennzeichnet, ist man noch waniger berechingt, z. B. aus dem kolloidchemischen Verha!ten der Stärke allein Schlüsse auf dasjenige des ganzen Michles zu ziehen. Es war daher den Verfassem klar, dab sie von vornherein nicht isolierte Bestandteile, sontern das Mehl unmittelbar selbst für die viskosimetrische Untarsuchung heranziehen mubren. Das Arbeiten wit ainem so komplizierten chemischen wie physikalischen Gemisch erscheint vielleitht von rein chemischen Gesichtspunkten aus bedenklich, nicht jedoch vom physikalischen, kolloidchemischen und besonders praktischen Standpunkt. Es schisebt ja überdies die spätere weitere Untersuchung der Viskosimetrie der Einzalbestanditelle des Mehles nicht aus.

$\mathrm{Da}$ viskosimetrische Untersuchungen uber Mehl-Wasser-Gemische bisher anscheinend nicht veröffentlicht worden sind, muBten die Verfasser selbst (mit ziemlichem Zeitaufwand) die Methodik entwickeln, bis sie so einfach und

- Es soi an die Arbeiten 2 . B. von E. Fouard und besonders yon $M . S$ an e erinnert.

4) Der ene Verasser (H. L tiers) wird demndchst in einer besonderen Abhandung in der "KotloidZaitschrift" Wber Versuche in deser Rlching Mitteilung nachen. 
eindeutig war, daf sie auch z. B. kleineren bäckereitechnischen Laboratorien empiohlen werden konnte. AeuBerlich läbt sich diese Entwicklung kerinzeichnen dadurch, das im Laufe der Untersuchung immer grobere Viskosimeter und entsprechend inmer konzentriertere Mehl-Wasser-Gemische angewendet wurden. Die inneren Gründe für diese Aenderungen sind folgende:

Wie erwahnt und loc cir. wher ausgefuhrt, ist das Mehl ein Polydispersoid, enthait m. $a$. W. bei Aufschwemmung in Wasser Tellchen auberordentlich verschiedener Gröbe und dazu noch von verschiedenem Lösungs- und Solvatationsvermögen. Bei allen Mehlen, ganz besonders aber bei den kleiereichen Kriegsmehlen findet sich nun ein grobdisperser Anteil " der aus Stäkekörnern und Kleietellchen zusammengesetzt und so grob dispers ist, dab er unter dem Einfiub seiner Schwere spontan sedimentiert. Eine, Aufschwemmung z. Bon 0,1 Proz. Mehl in kaltem Wasser labt in kurzer Zeit cinen groben Teil der dispersen Phase als Bodensatz fallen. Er. hitzt man nun ein solches Mehl-Wasser-Gemisch, so verkleistert zwar die Stärke und sedimentiert nicht oder nicht erheblich mehr, wohl aber fatlit das gleichzeitig geronnene Mebl-Riwe $\mathrm{B}$ neben den Kleieteilchen immer noch aus. Man hat es also in beiden Fällen, bei Verwendung sehr verdunuter Teiglösungen oder verdünter Mehlkleister, mit teilweise grobdispersen instabilen Systemen zu tun. Will man die Viskosität dieser Systeme mit dem Kapillar-Viskosimeter messen, so ist es offenber für cas Resultat von grober Wichtigkeit, we grof die Sedimentations-Geschwindigkeit ist gegenüber der $\mathrm{D}$ u c chlaufsGeschwindigkeit, welch letztere ja das Mah der Viskosität darstelit. Nur wenn die Sedimentations Geschwindigkeit s e $\mathrm{rkl}$ e in ist $\mathrm{gegen-}$ iz ber der Durchlaufs-Geschwindigkeit, ist es theoretisch wie praktisch möglich, einwandfreie Resultate zu erhalten. Ist die SedimentationsGeschwindigkeit von gleicher Grobenordung oder gar gröber als die Durchlauf-Geschwindigkeit des benutzten Viskosimeters, so treten auBerordentlich komplizierte Verhältnisse auf, die Resultate verschiedener Viskosimeter sind unter einander nicht mehr vergleichbar, es ergeben sich ganz unerwartete Abhängigkeiten der Durchlasfszeit von der Fullungshohe des Viskosimeters usw. 5 ).

5) Einzelheiten werden weiter unten gegeben
Die zunächst benutzten Viskosimeter nach Wilh. Ostwald hatten nun sämtlich zu enge Kapillaren, d. h. zu lange AusfluBzeiten, um ein bequemes Arbeiten mit Mehl-Wasse:-Gemischen zu gestatten. Zwar ist es, wie weiter untex gezeigt werden wird, in gewissen Fällen durchaus möglich, mit den üblichen Viskosimetern reproduzierbare und regelmäßige Zahlen zu erhaiten. Doch ist das Anwendungsgebiet beschränkt auf nur werig ausgemahiene, kleiearme Mehle, die Lösungen müssen filtriert werden usw. Es wurden daher zwei Säzze von Viskosimeterröhren von der Firma R. Götze in Leipzig angefertigt, die zunehmend immer grobere Kapillaren und entsprechend kleinere Ausflubzeiten hatten. Auf diese Weise konnte das Gebiet der meBbaren Lösungen erheblich erweitert und die Messungen selbst verbessert und erleichtert werden. Schlieblich ergab sich als das beste, am vielseitigsten anwendbare und bequemste Instrument ein selbstangefertigtes Viskosimeter mit ganz grober "Kapillare", dessen Dimensionen weiter unten angegeben werden sollen.

In entsprechender Weise wurden zunächst sehr verdünte Teiglösungen untersucht, d. h. Gemische von Mehl mit kaltem Wasser mit einem Gehalt von 0,1 bis höchstens 1,0 Proz. luftrockenem Mebl. Um die Sedimentations-Geschwindigkeit der Gemische zi verringern, wurden sodann gleichzeitig mit groberen Viskosimetern $M$ ehlkleister* Lösungen angewandt, die durch Erhltzen der Teiglösungen in noch näher $z u$ beschreibender Weise hergestellt wurden. Die Konzentrationen dieser Mehlkleister-Lösungen erreichten höchstens 2,0 Proz. SchlieBhich gelangten mit dem selbstgebauten Viskosimeter konzentrierte Teiglösungen zur Untersuchung, d. b. Gemische, dle ähnlich wie in der Praxis mit kaltem Wasser angeteigt wurden und eineri Mehlgehalt bis zu ca, 20 Proz. besaben.

Das Versuchsmaterial wurde mit einiger Mühe z. $T$. aus dem Handel bezogen, z. T. auch aus der Samming der D. P. A. zur Verfügung gestellt. $Z$ u gan\% besonderem Danke sind die Verfasser der Kunstmühle G. Barth in L a uf bei Nümberg verpflichtet, die in entgegenkonmendster Weise zwölf verschiedene Mehlsorten zur Verfügung stellte. Ein besonders interessantes Mehl rou abnorm schlechter Back. fähigkeit verdanken wir Herm Bäckermeister Wioland in Freising. weteren. 
Im Folgenden sollen nun die Ergebnisse in zwei Gruppen geordnet wiedergegeben werden :

I. Versuche mit Mehlkleister-Lösungen,

II. Versuche mit Teiglosungen.

Entsprechend den Ausführungen von Abschnitt 2 wăre es natürlicher, die Versuche mit Teiglösungen denen an Mehlkleistern voranzustellen; die Teigbereitung geht ja auch in der Praxis dem Backprozeh yoraus. Wenn hier von dieser begrifflich besseren Reiherfolge abgesetuen wird, so beruht dies darauf, da die Versuche mit Mehlkleistern vor denjenigen mit Teiglösungen angestellt wurden, daB gieichzeitig aber in der genannten Reihenfolge die Versuchstechnik allmählich mehr und mehr entwickelt wurde. $\mathrm{Da}$ nun den Verfassern auch die Schilderung der Ueberwindung dieser experimentellen Schwierigkeiten nicht uninteressant erscheint in Hinblick darauf, das zhnliche polydisperse Systeme wohl auch anderweitig in der Technik und $z$. B. auch in der physiologischen Chemie auftreten, so wurde obige Anordnung des Versurchsmateriais für $z$ weckmäbiger gehalten als die begrifflich strengere.

\section{Versuche mit}

Meblkleister-Lösungen.

4. Vorbereitende Versuche. Beim Erhitzen einer Teiglösung auf $100^{\circ}$ wird bekanntlich die Starke verkfeistert und das Mehleiweiff zur Gerinnung gebracht. Wie der handgreifliche Unterschied zwischen Brot und Teig zeigt, Ist dieser Vorgang in praktischem Mahstabe mit einer ganz erbeblichen Visinositutszunahme verknüpt, deren Auftreten in kleiner m Masstabe auch beim Arbeiten mit verdünnten Mehl-Wasser-Gemischen erwartet werden konnte. Diese Annahme lieb sich ohne weiteres bestatigen.

Versuchsweise erbitzten wir eine 0,3 prozentige Teiglösung unter Vermeidung von Verdunstung (Stopfen mit Kapillarrohr) einige Minuten bis zum Kochen. Bei $20^{\circ}$ gemessen ergab sich ein Durchlaufswert von im Mittel

$$
811 \frac{\text { sec. }}{5} \text { gegenüber } 725 \frac{\text { sec. }}{5} \text { Wasserwert. }
$$

Es zeigte sich also ein deutlicher Viskositätsunterschied. Es wurden nun einige Versuchs reihen mit verdïnnten Mehlkleister-Lossungen angestell, um zunächst den Einflu der Konzentration auf die Viskosität kennen zu lernen. Um eine möglichst grohe Gleichmäkig. keit zu gewährleisten, warden die kalt herge. stellten Teiglosungen ? gleichzeitig eine Viertelstunde lang im kochenden Wasserbad verkleistert, gleichmäbig auf Zimmertenperatur abgekäht und damn bei $20^{\circ}$ viskosimetriert. Nachstehende Tabelle 1 enthält die Resultate, die gleich in rehativen Viskositäten (d. b. bezogen auf den Wasserwert als Einheit) wiedergegeben sind. In der dritten Spalte $\eta_{a}=\left(\eta-\eta_{0}\right) \times 1000$ finden sich die $Z u$ ahmen der relativen Viskosität in Tausendstel der Einbeit verzeichnet $^{7}$ ). Diese Zahlen geben viellercht ein besonders deutliches Bild der Viskasitatsvermehrung bei zunehmender Mehlkonzentration. - Selbstverstandlich sind alle Zahlen Mittelwerte aus mindestens drei Ablesungen.

Tabelle I.

Einflut der Konzentration auf die Viskosität verdinter Menlkleister-Lösungen von 60prozentigem Weizenmehl.

\begin{tabular}{c|c|c}
\hline$c$ & $\eta$ & $\eta_{\Delta}=\left(\eta-\eta_{0}\right) 1000$ \\
\hline Proz. & $\eta$ & 28 \\
\hline 0,1 & 1,026 & 67 \\
0,2 & 1,067 & 118 \\
0,3 & 1,118 & 180 \\
0,4 & 1,180 & 250 \\
0,5 & 1,250 & 475 \\
0,8 & 1,475 & 619
\end{tabular}

Die 0,8 und 1,0prozentigen Kleisterlösungen waren infolge hatufiger Verstopiung durch grobere Kleieteilchen schwer zu messen.

Da uns auch das $\mathrm{Nachmehl}$ von diesera 60 prozentigen Weizenmehl ${ }^{3}$ zur Verfugung stand, fühten wir auch rnit diesem eine varsuchsserve in der beschrieberen Weise ans, nut mit dem Unterschied, daf wir nach dem $\mathrm{Er}$

6) Die Herstelinng solcher verdünter Teiglösungen geschah folgendermafen: In einem Achatmorser warde die genat (auf drel Dezimalen) abgewogene Mehsmenge, z. B. $0,100 \mathrm{~g}$, mit kleinen Wassermengen verrieben und die erhattene Aufschwemmung durch ein Filter von feinen Metallmasselin filtrlert, um jene sehr groben Kleiepartikel zu enternen, die, wie sich bald herausstellte, das Viskosimeter in listigster Welse verstopfen. Die Aufschwemmung wurde aus den Mörser quantitativ herausgespiutt und im Me\&kolben 2. 3. auf $100 \mathrm{cen}$ aufgefillit. Weiteres über die Herstellung konzentrierter Teiglosungen siehe Abschnitt II.

7) Clierbel bedeutet of die Viskositżts z u n a h eq * die gemessene relative Viskrosităt des Kielsters, \%o die Viskosität des Wassers.

6) Prozentzanlea wor Mehlen bedeuten bekanntich thren Ausmahlungsgrad. Je niedriger die Prozerizahi, un so stärkereicher wnd kieieärmer ist das Mehl. Das Nachmehl entball die äbrigea Kombestandteile, in obigen Falle also sie restlichen $40 \mathrm{Proz}$. 
hirzen und Abkühlen die Lösung durch dea bereits erwánten Metallmusselin filtrierten. Da das Nachmehl bekanntich auberordentlich reich an gröberen Kleieteilchen ist, war diese Mabnahme nötig. Die unfiltrierte Kleisteriossung honnte wegen ständigen Verstopfens der Kapillare nicht gemessen werden. Es sel bemerkt, daR die vorn Metallfilter entfernten Kleieteilchen bequen mit tem bloben Auge erkennbar und daher als "makroheterogene Phase" jedenfalis nur von geringem Einflub auf die Viskosităt sind. Die erhaltenen Resultate finden sich in Tabelle II.

Tabelie II.

Einflug der Konzentration auf die Viskositat verdunnter Mehlkleister-Lösungen; Nach ne hle zum 60 prozentigen Weizenmehi der Tabelle l.

\begin{tabular}{c|c|c}
\hline$c$ & $\eta$ & $n_{0}=\left(\eta-\eta_{0}\right) 1000$ \\
\hline Praz. & $\eta$ & 22 \\
0,1 & 1,022 & 33 \\
0,2 & 1,033 & 64 \\
0,4 & 1,064 & 106 \\
0,8 & 1,106 & 191
\end{tabular}

Bede Varsuchsrethen sind in Fig. 1 graphisch dargestellt. Man erkennt sinnfallig, wie der grobe Unterschied in den chemischen und vor

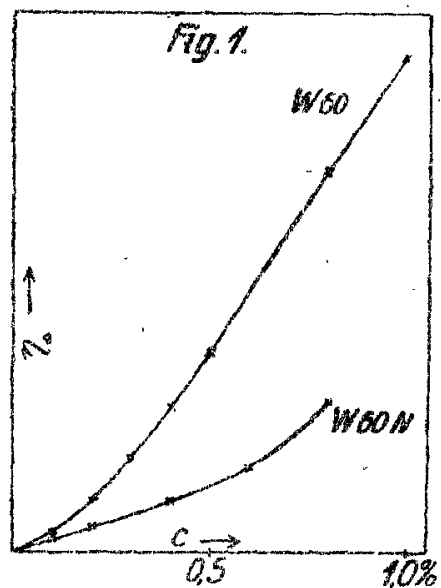

allen Dingen backtechnischen Eigenschaften dieser beiden Mehle sich auch im Verlaufe der Viskositäts -Konzentrationskuryen äußert.

5. Reproduzierbarkeit der $Z$ ahlen und hiermit zusammenhängende Fragen. Nachdem sich herausgestellt hatte, dah auf diese Weise in erster Annäherung ein praktisch durchfuhrbares Verfahren gefunden worden war, wurde der Einflut verschiedener Faktoren auf die Versuchstechnik mäher studiert, um festzustellen, ob sich die Methode auch für feinere Differenzen und unter stärker varjiérenden Bedingungen gebrauchen lieb.

a) Zur Feststellung der Reproduzierbarkeit der Viskositătswerte wurden folgende Versuche angestelit: Zweimal je 1,000 g Mehl wurden wie oben geschildert mit $100 \mathrm{ccm}$ Wasser in kochenden Wasserbad verkleistert und nacheinander viskosimetriert.

Wasserwert: $\left.722,722,724,7233^{9}\right) ; \eta_{0}=1,000$.

Probe l. 1,0prozentiger Mehlkleister: $1287,1289.1284 ; \eta=1,779$.

Probe II. 1,0 prozentiger Mehlkieister: $1272,1276,1273 ; \eta=1,761$.

Die Abweichung betrug also 1,0 Proz.

Ein zweiter Versuch mit 0,5 prozentigem Kleister ergab bei einem anderen Viskosimeter:

Wasserwert: 739 .

Probe 1. 0,5 prozentiger Mehlkieister: $838,837,836,840,838,837,839$; Mittel $=838 ; \eta=1,134$.

Probe II. 0,5 prozentiger Mehlkleister: $826,824,827,822,825,823,824$; Mittel $=824 ; \eta=1,115$.

Die Abweichung betrug in diesem Palle also 1,7 Proz. Die Versuche zeigen, dab die Versuchstechnik gestaltet, die Resultate bei ein und demselben Mehl mit einer Abweichung von $1-2$ Proz. wieder zu erhalten.

b) Ein weiterer Versuch solite über den Einflub der Nachquellung orientieren, d. h. über die evtl. Viskositätszunahme, die durch das allmähliche Auflösen und Aufquellen der Mehleiweißstoffe zu erwarten war. Es wurden daher wie in voranstehendem Versuch $0,500 \mathrm{~g} \mathrm{Mehl} \mathrm{mit} \mathrm{etwas} \mathrm{Wasser} \mathrm{angeteigt,}$ jedoch erst nach 14 stündigem Stehen auf $100 \mathrm{ccm}$ aufgefüllt und weiterhin in oben beschriebener Weise verkleistert. Es ergab sich eine Viskosilat $(\eta)$ von 1,172 gegenüber 1,125 ,

dem Mittel der voranstehenden Messungen für Kleister gleicher Konzentration und sonst gleicher Behandlung. Es zeigt sich also eine deutliche Viskositätszunahme infolge der Nachquellung, und zwar in vorliegendem Beispiel um über 4 Proz. bezogen auf $\eta$ oder um $41,6 \mathrm{Proz}$. bezogen auf $\eta_{\Delta}$.

c) Es ist aus Viskosimetrie anderer, einfacherer bydratisierter Emulsoide bekannt, das man keineswegs die gleichen Viskositätswerte bei

2) Diese sowie die folgenden Durchlaufszeiten bedeuten immer Funftel-Sekunden. 
gleicher Kolloidkonzentration erhält, wenn man einmal die Lösungen mittelst $A$ bwi egen, das andere Mal mittelst $\mathrm{V}$ erdünnen einer konzentrierien Stammlösung herstellt. Um festzu. stellen, ob analoge Verhältnisse auch beim Mehlkleister vorliegen, wurde folgender Versuch angestellt:

Probe II des einprozentigen oben erwähnten Kleisters wurde auf die Hälte verdünnt. Sofort nach der Verdünnung gemessen ergab sich $\eta=1,215$,

d. h. ein Wert, der gegenubber dem unter a) erhaltenen Wert des 0,5 prozentigen Kleisters von durchschnittlich ${ }^{\circ} 1,125$ erbeblich, nämlich um 7,2 Proz. $z u$ hoch ist.

Nachdem die Probe $2-3$ Stunden im Viskosimeter belassen worden war, wurde ihre Viskosität erneut gemessen. Jetzt ergab sich: $\eta=1,135$

d. h. ein Wert, der dem durch $A$ bwiegen erhaltenen Viskositätswert (Mittel: 1,125) ganx wesentlich näher steht. Es geht hieraus her vor, dab bei unmittelbarem Verdunnen eines konzentrierten Mehlkleisters die Losurigen zunăchst eine in ö h e re Viskosität zeigen als wenn sie durch Abwiegen hergestellt worden waren, daf aber im Laufe einiger Stunden eine deutliche Annäherung des ersten zu hohen Wertes an den Viskositătswert der abgewogenen Gemische festzustellen ist. Die Erklärung für dies Verhalten berwht jedenfalls darauf, daf in einem konzentrierten Mehlkleister gröBere Teilchenaggregate vorhanden sind, die sich beim Verdünnen erst $\mathrm{a} 1 \mathrm{~m} \mathrm{~m}$ hlich in kleinere, der Verdünnung entsprechende Aggregate zerteilten.

d) Daß solche zeitliche Verinderungen der Viskositat, die teilweise auf kolloidphysikalische Erhöhungen des Dispersitătsgrades, teilweise auch auf die spontane Dehydratation der gequollenen Teilchen zurückzuführen sind, in erheblichem Ausmaie eintreten können, zeigt folgender Versuch: Ein frisch bereiteter Mehlkleister (ca.0,5 Proz.) zeigte unmittelbar nach der Herstellung eine Durchlaufszeit von 1060 Fünftel-Sekunden, nach 14 stündigem Verweilen im Viskosimeter 1017, nach weiteren zehn Stunden nur noch 920, wobei alle Zahlen das Mittel mehrerer Ablesungen sind. Es sind beim Altern des $\mathrm{Kle}$ isters ${ }^{13}$ ) Abnahmen der Viskosität die Regel; Zurnahmen treten nur selten und unregelmaBig aut. Parallel mit dieser spontanen Abnahme der Viskosität geht eine interessante Aendenung der $\mathrm{Sch}$ a $\mathrm{w}$ m-

19) Für Teiglósungen gilt diese Regel keineswegs, wie aus Abschnitt II hervorgehen wird. fähigkeit des Meblkleisters. Um für gute Durchmischung zu sorgen, ist es bekanntlich zweckmäßig, vor der Messung einige Luftblasen durch die Lösung in Viskosimeter zu schicken. Bei frisch hergestellten Mehlkleistern ist dieses Durchblasen nun von heftiger, zuweilen sehr lästiger Schaumentwicklung begleitet. Je alter die Kleisterlösungen werden, um so geringer wird diese Schaumfähigkeit.

6. Weitere Entwicklung der Methodik. Einflub der Sedimentation. Trotzdem es auf die geschilderte Weise möglich war, in erster Annăherung konstante Resultate und ziemlich regelmäBige Kurven zu erhalten"), befriedigte die Methodik nicht auf die Dauer. Aus oft unbekannten Gründen ergaben sich plötzlich zwischen den einzelnen Ablesungen erhebliche Differenzen, zuweilen wiesen diese Differenzen einen deutlich periodischen Gang auf, nur bei überaus peinlicher Gleichmatigkeit in der Herstellung der Mehikleister waren die Resultate reproduzierbar, insbesondere aber zeigten sich bei den sehr verdunnten Kleisterlösungen häufig auffällige UnregelmäBigkeiten, die $z$. B. ganz unregelmäBige Anfangsteile der Konzentrations-Viskosităts-Kurven ergaben.

Es ist aicht unsere Absicht, die große Anzahl yon Versuchen im Einzelnen zu schildern, die wir zu dem Zwecke anstellten, die Ursachen dieser Unregelmäbigkeiten, namentlich bei den stark verdünnten Kleisteriösungen herauszufinden. Wie bereits erwähnt, ergab sich als Hauptursache die Instabilität bzw. die spontane Sedirnentation eines Teiles der dispersen Phase, genauer ausgedrückt, das Verhältnis dieser Sedimentationsgeschwindigkeit zur AusfluBgeschwindigkeit des Viskosimeters. Folgender Versuch zeigt, wie dieser Foktor in dex Tat bei den verdunnten MehlkleisterLösungen wesentlich wichtiger ist als bei den konzentrierteren.

a) Eine Reihe von Reagenaröhren wurde mit glefchen Mengen von Kleisterlösungen steigender Konzentration beschickt und im Reagenzglasgestell zur Beobachtung der Sedimentation nebeneinander gestellt. Alle Röriren wurden naturlich gleichzeitig zu Beginn des Versuchs

ix) Es sei angemerit, daß sich z. B. die Zahlen von Tabelie I recht gut berechnen lassen, entsprechend den Formein $\eta=\eta_{0}+K, C n(K=25,66 ; n=1,4)$ oder $\log \eta=K_{2}\left(c-K_{2}\right)$, wobei $K_{1}=0,23_{3} K_{2}=0,07$ ist; die mittlere Abweichung zwischen Beobachturg und Rechnung beträgt im ersteren Falle 0,4 Proz., im zweiten 0,7 Proz. 
geschütrik, Die Konzentrationen bewegten sich zwischen 0,1 und 2,0 Proz.

Nach arei Minuten zeigtem samtiche Röhren bis einschlieblich derjenigen mit 0,6 Proz. einen Bodensatz, und zwar setzten die 0,4- und 0,6 prozentigen Lòsungen nicht wesentlich mehr $a b$ als z. B. der 0,3 prozentige Kleister. Die Lösungen höherer Konzentration erschienen alle noch homogen.

Nach fün: Minuten erschien wich in dem 0,8 prozentigen Kleister etwas Bodensatz. Die verdunnteren Losunger hatten noch mehr abgesetzt.

Nach 15 Minuten erschien auch im 1,2 prozentigen Kleister ein schwacher Bodersatz.

Nach 45 Minuten war in allen Rohren, auch in denjenigen hoherer Konzentration ein ausgesprochener Bodensatz zu sehen mit Âusnahme des 1,2 prozentigen Kleisters, der ohne jede sichtbare Veränderung geblieben war.

Es gehi hieraus hervor, dat erstens die Sedimentationsgeschwindigkeit in den verdunnteren Kleisterlösungen von ansehnichem absolutem Betrage ist, insbesondere aber von derselben Gröbenordnung die Durchlaufszeit der berutzten Viskosimeter (etwa zwei Minuten). Ferner zeigt sich, daB die Sedimentationsgeschwindigkeit von der Konzentration des Kleisters abhängt derart, daß sie bei den höchsten Konzentrationen bemerkenswerter Weise am kleinsten ist, während sie in den niedrigsten Konzentrationen bis etwa 0,5 Proz. sehr grob und wenigg abhängtg von der Konzentration zu sein scheint. Der Grund für diese grobere Instabilitä der verdünnten Lösungen liegt wobl darin, das erst bei höheren Mehlkonzentrationen der Kle:ster jene $\mathrm{z} u \mathrm{~s}$ a $\mathrm{m}$ m e $\mathrm{n}$ hängen de mechanische Beschaffenheit annimmt, wie sie für Gallerten kennzeichnend ist. - Es ist einleuchtend, dab diese Sedimentation namentlich der verdünnteren Lösungen die Messungen ihrer Viskosität in der bisher beschriebenen Art erheblich stören muBte.

b) Aber auch quantitativ konnte gezeigt werden, da in den verdünnten Kieisterlosungen tatsächlich die sedimentierenden grober dispersen Anteile für die erwähnten Unregelmasig : keiten verantwortlich $z$ u machen waren. Es wurden zwei Serien von Kieisterlösungen verschiedener Konzentration in der beschriebenen Weise hergestellt. Die eine Serie wurde-nur durch Metallmusselin, die andere dagegen durch ein gewöhnliches Faitenfilter filtriert, wobei in letzterem Falle das Filtrat nochmals zurückgegossen purde. Kleisterlbsunger von mehi als 0,5 Proz. konnten nicht mekr flitriert werden.
Folgende Tabelle III und Fig. 2 zeiger die erhaltenen Viskositätswerte.

Tabelle lll.

Einflutes filtrierens

auf die Viskositat verdünter

Mehlkleister-Lostungen

(9)prozentiges Weizenmebl).

\begin{tabular}{|c|c|c|c|}
\hline \multicolumn{2}{|c|}{$\begin{array}{l}\text { a) Filtriert durch } \\
\text { Metallmusselin }\end{array}$} & \multicolumn{2}{|c|}{$\begin{array}{l}\text { b) Filtriert durch } \\
\text { Papierfilter }\end{array}$} \\
\hline $\begin{array}{c}\text { c } \\
\text { Zrou. }\end{array}$ & n & $\begin{array}{c}c \\
\text { Proz. }\end{array}$ & $\eta$ \\
\hline $\begin{array}{l}0,1 \\
0,2 \\
0,3 \\
0,4 \\
0,5 \\
0,6 \\
0,8\end{array}$ & $\begin{array}{l}1,105 \\
1,184 \\
1,235 \\
1,287 \\
1,334 \\
1,456 \\
1,514\end{array}$ & $\begin{array}{l}0,1 \\
0,3 \\
0,4 \\
0,5\end{array}$ & $\begin{array}{l}1,0025 \\
1,0119 \\
1,0190 \\
1,0310\end{array}$ \\
\hline
\end{tabular}

Besonders die graphische Darsfellung ${ }^{13}$ ) zeigt deullich, dab der durch Papier von den sedimentierenden Anteilen befreite Kleister auch

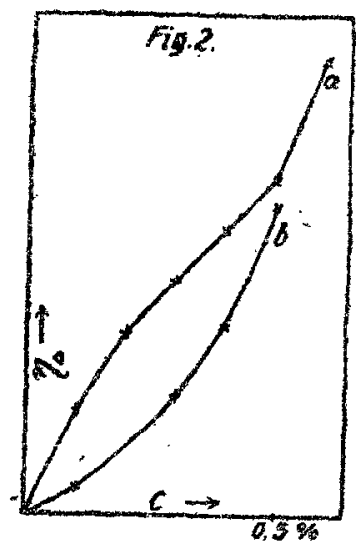

bei grofen Verdinnungen in völlig regelmäBiger Abhängigkeit von der Konzentration steht, während der nur durch Metallmussein gegangene Kleister in diesen Verdünaungen durchgängig viel zu hohe Viskositätswerte aufweist. Bei hoheren Konzentrationen, z. $\mathbf{B}$. schon von etwa 0,5 Proz. ab, bel denen die Sedimentationsgeschwindigkeit, wie gezeigt, wesentlich kleiner ist, schließen sich auch die Werte des nicht durch Papier gegangenen Kleisters der normalen Konzentrationskurve wieder an.

c) Zur Verbesserung der Methodik wurden zwei Wege gleichzeitig eingeschlagen. Einmal wurden neue Viskosimeter verwendet mit

19) Der Deutfichkeit wegen sind die Zahien des durch Fapler fiftrierten. Klelsters in zehnmal groberem Matstabe in die Figur eingetragen worden. 
gröheren Kapillaren, andererseits wurde durch eine bessere Verkleisterung die absolute Visko. sität der Versuchslösungen erhönt, um auf diese Weise die Sedimentationsgeschwindigkeit herabzusetzen. Es gelang so in der Tat, bessere Resultate 2 w erhalten und besonders die Fehler bei den großen Verdünnungen einzuschränken.

Zur Erzielung einer besseren Verkleisterung erschien es zweckmälig, die Versuchstechnik näher an das bekannte Verfahren anzuschließen, nach dern in der Praxis möglichst "stefie ${ }^{\prime}$ Kleister hergestellt werden. Stalt das mit Wasser aufgeschwemmte Mehl nachträglich zu erbitzen, wurde zundchst bei einem orientierenden Versuch $0,5 \mathrm{~g}$ Mehl mit seht wenig Wasser angerührt und dann unter Nachspülen und Umrühren in kieinen Portionen in $75 \mathrm{ccm}$ kochendes Wasser gegossen. Unter annaherndem Ersatz des verdampfien Wassers wurde genau eine Viertelstunde auf dem Drahtretz weitergekocht, abgekuhlt und auf $100 \mathrm{ccm}$ ergänzt.

Gleichzeitig wurde ein 0,5 prozentiger Mehlkleister nach der bisherigen Methode hergestellt.

Die Durchlaufszeit des nach dem neueq Verfahren hergestellten Kleisters betrug im Mittel 1058, diejenige des nach dem alten Verfahren hergesteliten 901. Es ergibt sich also eine Zunahme von über 17 Proz.

d) Um festzustellen, wie lange erhitzt werden muBte, um annähernd das Maximum der Viskosirät bei der Verkleisterung zu erzielen, wurde folgender Versuch angestellt: Ein 0,3 prozentiger Kleister, der in oben beschriebener Weise hergestellt worden war, wurde mit KapllarrohrVerschluf weiter in kochendem Wasserbade erhitzt. Nach verschiedenen Zeiten wurden Proben entnommen und nach Abkühlung unter sorgfältiger Vermeidung von Verdampiung viskosimetriert. Es ergaben sich folgende Durchlaufszeiten (Mittelwerte):

$1 / 2$ Stunde im Wasserbade rtaitzt: 1098

1 " " " " ; 1085

2 Stunden.. $\quad$ "

Das Vishositätsmaximum ist also wahrend der ersten halben Stunde zweilellos erreicht.

Zur Demonstration der Reproduzlerbarkeit wurde sodann ein zweiter Versuch in genau der gleichen Weise mit $0 ; \overline{5}$ prozentigem Kleister $(0,600 \mathrm{~g}$ Mehl auf $200 \mathrm{~g}$ Wasser aurgewogen) ausgeführt. Nach halbstündigem Erhitzen im Wasserbade ergab sich eine mitulere Durchlaufszeit von 1099. Die Uebereinstimmung mit obigem Wert nach entsprechender Zeit ist also ausgezeichnet. e) Auf Grund dieser und anderer methodischer Versuche ergab. sich schlienlich das folgende Verfahren, das sich ausgezeichnet bewahr hat und ohne Aenderung von uns bei allen weiteren Versuchen über die Vishosität von Mehlkleistern angewandt wurde:

Die auf drei Dezimalen genau abgewogere Menge Mehl wird in einen kleinen Bechergiäschen von $50-100 \mathrm{ccm}$ Inhalt mit $10 \mathrm{ccr}$ Wasser derart eingeteigt, das man zuerst nur etwa $2-3 \mathrm{ccm}$ Wasser zusetzt, einen homogenen Teig damit anrührt, der frei von Klimpchen sein muk, und dann mit dem Rest der 10 ceni Wasser verdinnt. Es gelingt, wie bekannt, auf diese Weise viel besser, eine klümpchenfreie Lösung zu erhalten, als wenn man gleich auf einmal die gesamte Wassermenge zugibt. Unterdessen hat man in einem 300 bis $400 \mathrm{ccm}$ fassenden Becherglas $100 \mathrm{ccm}$ Wasser zum Kochen gebracht. In das lebhaft kochende Wasser gieft man nun die gut aufgerïhrte Mehllösung ein und hält die Flüssigkeit moglichst nahe am Sieden. Mit zweimal le $7-10 \mathrm{ccm}$ Wasser wird das kleine Becherglaschen unter Zuhilfenahme eines Gummiwischers quantitativ in das grobe Becherglas übergespült und dann der Kleister unter Umrohren noch etwa eine Minute im Kochen erhalken (Vorsicht wegen Schäumens!). Die beilbe Lơsung wird in einen tarierten $500 \mathrm{ccm}-$ Erlenmeyerkolben filtriert, wobei man durch Abheben des Musselins von der Trichterwand und Aufwirbein der Plüssigkeit die etwa stockende Filtration beschleunigt. Mit heibem destilliertem Wasser wird in zwei Portionen von $10 \mathrm{ccm}$. und mit Zuhilfenahme des Gummiwischers das Becherglas quantitativ ausgespult; die Waschwässer werden auch durch das Filter gegossen. Dann spritzt man mit heißem Wasser in kräftiger Strahl das Filter vom Rand an nach, unter offterem Aufwirbeln und Abspritzen des Filterinhalts. Der Erlenmeyerkolben wird sodann auf einer guten Tarierwage auf $200 \mathrm{~g}$ aufgefüllt. Hierauf wird der Kolben mit einem gut schliefenden Gummi- oder Korkstopfen verschlossen, der ein etwa $15-20 \mathrm{~cm}$ langes Kapillarglasrohr trägt. Kolben mit Inhalt werden darauf noch eine halbe Stunde im Wasserbade erhitzt, um kleine UnregelmäBigkeiten, bẹi der Verkleisterung auszugleichen. Sodann wird unter der Wasserleitung oder mit Eiswasser abgekihlt und bis zur Messung der Kolben oder ein Teil des Inhaltes in ein Wasserbad von $20^{\circ}$ gestelit. 
Die Beschreibung dieser Versuchstechnik wurde so ausführlich gegeben, da dies Verm fahren das Resultat mannigfaltiger Vorversuche ist und sich, wie gesagt, durchaus bewahte bet. Ueberdies ist seine Ausführung nach einiger Uebung weniger kompliziert als die Beschreibung vielleicht vermuten läkt.

f) In bezug auf das geeignetste $V$ is k osimeter ergab sich, das die besten, insbesondere gleichmätigsten Werte erhalten werden, wenn man möglichst grobe Kapillaren verwendet. Natürlich kann man in dieser Richtung nicht allzuweit gehen, da bei zu groben Kapillaren die Durchlaufszeiten kieiner und die Messungen infolgedessen aus diesem. Grunde ungenauer werden. Geeignet sind $z$. B. Viskosimeter. röhren, die bei einem Durchlaufsvolum von $5-10 \mathrm{ccm}$ eine Durchlaufszeit von höchstens ein bis zwei Minuten für Wasser baben; Röhren mit noch kleineren Wasserwerten sind vorzuziehen, wenn es sich z. B. um die Messung von hoch ausgemahlenen, sehr kieie reichen Mehlen handelt.

Von grober Wichtigkeit ist es, auf eine sehr gleichmäbige Füllung des Viskosimeterrohres zu achten, wie dies ja schon aus der Theorie des Kapillarviskosimeters folgt. Da das optimale Volum der einzufîllenden Flüssigkeit aicht immer eingr runden Zabl von ccm entspricht, die man mittelst Pipette eimhühren konnte, so ist am einfachsien, am breiten Schenkel des Viskosimeterrohres eine Marke anwubringen, bis 7u der man die Plässigkeit einfüllt.

Ueber die besten Dimensionen eines Viskosimeters zur Messung honzentrierter Teiglösungen wird weiter unten noch berichtet werden.

\section{Reierate.}

\section{Arbeiten über allgemeine Kapillarphysik.}

$\mathrm{K} b \mathrm{nig}$, W., Der Streit un das Elektroti. (Die Naturwiss. 5, 373, 1917.)

Der Kolloidchemiker ist auch deshaib in hohem Grade an den Meinungsverschiedenheiten interessiert, die hier in ubersichtlicher Weise zusammengestelit sind, weil die Untersuchungsmethoden meist koiloidchemischer Art sind. Und zwar betreffen sie das Ver halten sehr disperser Flüssigkeiten und fester Stoffe in Gasen. Das Eingehen auf dieselben erspart eine ganze Reihe von Einzelreferaten.

Es kam darauf an, festzustellen, ob auch bei der Elektrizitatsleitung in Gasen die von den materielien Teilchen ibertragenen Elektrizitäsmengen in gleicher Weise Elementarquanten erkennen liefsen wie diejenigen bei der Elektrolyse von Flissigkeiten.

Townsend and J.J. Thomson maßen die Gesamtladung, die eine gewlsse lonenmenge mil sich führt, und dividierten sie durch die Zahl der Ionen. Letztere bestimmten sic, inden sie die lonen als Kondensationskerne in einer durch adiabatische Ausdebnung wasserubersättigten Luft benutzten. Aus der Geschwindigkeit des Niedersinkens der gebildoten Nebelwolke konnte nach der Formel von S tokes die Größe der Nebeltröpfchen berechnet werden. Die dann folgende Bestimimung der Tröpfchenzahl war die ubliche.

H. A. WiIs on brachte an dem Nebelgetă einen elektrischen Kondensator an, dessen Kraftlinien genau vertikal standen, und bemas den Grad der Expansion so, das sich der Nebel nur auf den negativen lonen bildete. Indem er dann die Gesthwindigkeit des Sinkens der Nebelwolke nur unter dem Einflub der Schwerkraft, das andere Mal dann mab, went ein elektrisches Feld der Schwere entgegenwirkte, konnte et das Verhältuis der Ladung der Troptchen zu ihrer Masse bestimmen.

Aus diesen Untersuchungen war noch nicht $z$ t erkennen, ob jedes einzelne Ion die gleiche Ladung trage, oder ob der erzielte Wert nur der Mittelwert aus vielen sehr ungleichartigen, teils gröberen, teils kleineren Werten sei. Deshalb waten Messungen an einzelnen lonen nötig. Millik an gelang es nicht, durch Steigerung des elektrischen Feldes die Wolke als Ganzes schwebend zu erhalten. Vielmeht worden dabel die dispersen Wasserteilchen teils nach oben, teils nach unten an die Kondensatorplatten herangerissen. Einzelne Teilchen blieben jedoch schweber, und an diesen war die Individualbeobachtung möglich. Stets ergab sich eine ladung, die das Vielfache von 4,65 . 10-10 war.

Un die störende Vergasung der dispersen Teilchen zu verhindern, ersetzte et spater das Wasser durch Glyzerin, Oel oder Qnecksiber. So wurde eine nehrstiandige Beobachtung eines einzelnen schwebenden Teilchens möglich. Beí den Berechnungen erwies sich eine Korrektur der Stokes'schen Formel nottig, weil diese in ihrer einfachen Form nicht mehr giltig ist, wenn der Durchmesser des dispersen Teilchens von der Gröbenordung der mittleren Weglänge der Gasmolekilie ist. Aus seinen neuesten Messungen (1916), bei denen er bis zu einem Tropfendurchmesser von $1,25 \cdot 10^{-5} \mathrm{~cm}$ hinunterging, ergab sich eine Elementarladung von 4.774 . 10-10. Dabei konnte er auch feststellen, das die Oeltröptchen sich wie feste Kugeln verhalten, daB ilare Dichtigkeit von der des gewohnlichen Deis kaum verschieden ist, und daB der Reibungswiderstand durch die Ladung der Teilchen nicht verändert wird.

Ehrenhaft ging von seinen Untersuchungen iber die Brow n'sche Bewegung yon Metallteilchen in Gasen aus, die er aus einem zwischen Metallelektroden bremnenden Lichtbogen in die Kammei des Ultramikroskops hineingesaugt hatte. Auch er fuhrte. damn einen Kondensator in die Rauchkammer ein. Als Metalle kamen $\mathrm{Au}, \mathrm{Ag}$ und $\mathrm{Pt}$ zut Verwendung. Solange die Teilchen die Grobe der von Mllilka benutzten hatten, ergab sick auch ein entsprechender Ladungswert. Dagegen zeigten sich geringere Làdungen, wenn der burchmesser nur "/, oder $1 / \%$ wurde. D. Konstantinows 